\title{
Konventionelle Labordiagnostik der Hämophilie B II. Diagnostik der Hämophilie-B-Konduktorinnen
}

\author{
Conventional Laboratory Diagnostics of Haemophilia B \\ II. Detection of Haemophilia-B-Carriers \\ G. Lutze', K.-J. Hartung', H.-J. Presser', H. Urbahn' \\ 'Institut für Klinische Chemie und Laboratoriumsdiagnostik der Medizinischen Akademie Magdeburg
2 Walther-Fricdrich-Krankenhaus Magdeburg
}

\section{Zusammenfassung:}

19 genetisch sichere und 46 genetisch mögliche Konduktorinnen der Hämophilie B wurden mehrfach gerinnungsanalytisch untersucht. Die sicheren Konduktorinnen wurden einer Hypo-IX-ämie und einer Dys-IX-ämie (Normodys-IX-ämie bzw. Hypodys-IX-ämie) zugeordnet. Bewertungskriterien bei der Beurteilung des Konduktorinnenstatus waren IX:C, IX:Ag und der Quotient IX:C/IX:Ag.

Bei genetisch sicheren Konduktorinnen ergaben sich folgende Quotienten:

1. Hypo-IX-ämie um $1,0(0,70-1,16)$ IX:C/IX:Ag

2.1 Normodys-IX-ämie um $0,5(0,19-0,69)$

2.2 Hypodys-IX-ämie entsprechend 1. oder

entsprechend 2.1

Bei genetisch möglichen Konduktorinnen waren diese Ergebnisse die Grundlage für die Zuordnung zu einem Konduktorinnenstatus.

Die konventionelle Konduktorinnendiagnostik wird als eine sinnvolle Diagnostikform angesehen, die der genomischen Diagnostik vorgeschaltet sein sollte. Sie ist schnell und einfach durchführbar und macht bei einer Anzahl von Frauen weitere Untersuchungen überflüssig.

Schlüsselwörter:

Hämophilie B - Konduktorinnendiagnostik - Faktor-IX-Aktivität - Faktor-IX-Antigen

\section{Summary:}

19 obligatory and 46 possible female carriers of hemophilia B were hemostaseologically investigated several times. Obligatory carriers were subdivided in Hypo-IX-emia and Dys-IX-emia (Normodys-IX-emia or Hypodys(X-emia).

Criteria of carrier state were IX:C, IX:Ag and the quotient IX:C/IX:Ag.

Obligatory carriers have shown the following results:

1. Hypo-IX-emia

IX:C/IX:Ag

2.1 Normodys-IX-emia about $0.5(0.19-0.69)$

2.2 Hypodys-IX-emia corresponding to 1. or 2.1

Possible carriers of haemophilia B were classified by comparing their results with them of obligatory carriers. The hemostaseological examination should be used as basis for genomic diagnostics. The methods are quickly and easily to perform and allow in several cases of carriers to avoid genomic diagnostics.

\section{Keywords:}

Hemophilia B - detection of carriers - factor-IX-activity - factor-IX-antigen

\section{Einleitung}

Eine genetische Beratung von Frauen aus Hämophilie-BFamilien und die Veranlassung gezielter Maßnahmen ist nur möglich, wenn' eine eindeutige Identifizierung der Konduktorinnen vorgenommen werden kann (1-3).

Genetisch sichere Konduktorinnen sind auch ohne Labordiagnostik als solche zu ermitteln. Es sind dieses
1. Töchter eines Hämophilen,

2. Mütter von mehr als 1 hämophilen Sohn bei separaten Geburten,

3. Mütter von 1 hämophilen Sohn bei mindest 1 hämophilen Verwandten in der mütterlichen Linie. 


\section{Enzygnost巴 Anti-HIV 1/-HIIV 2}

\section{E[tappensieger!}

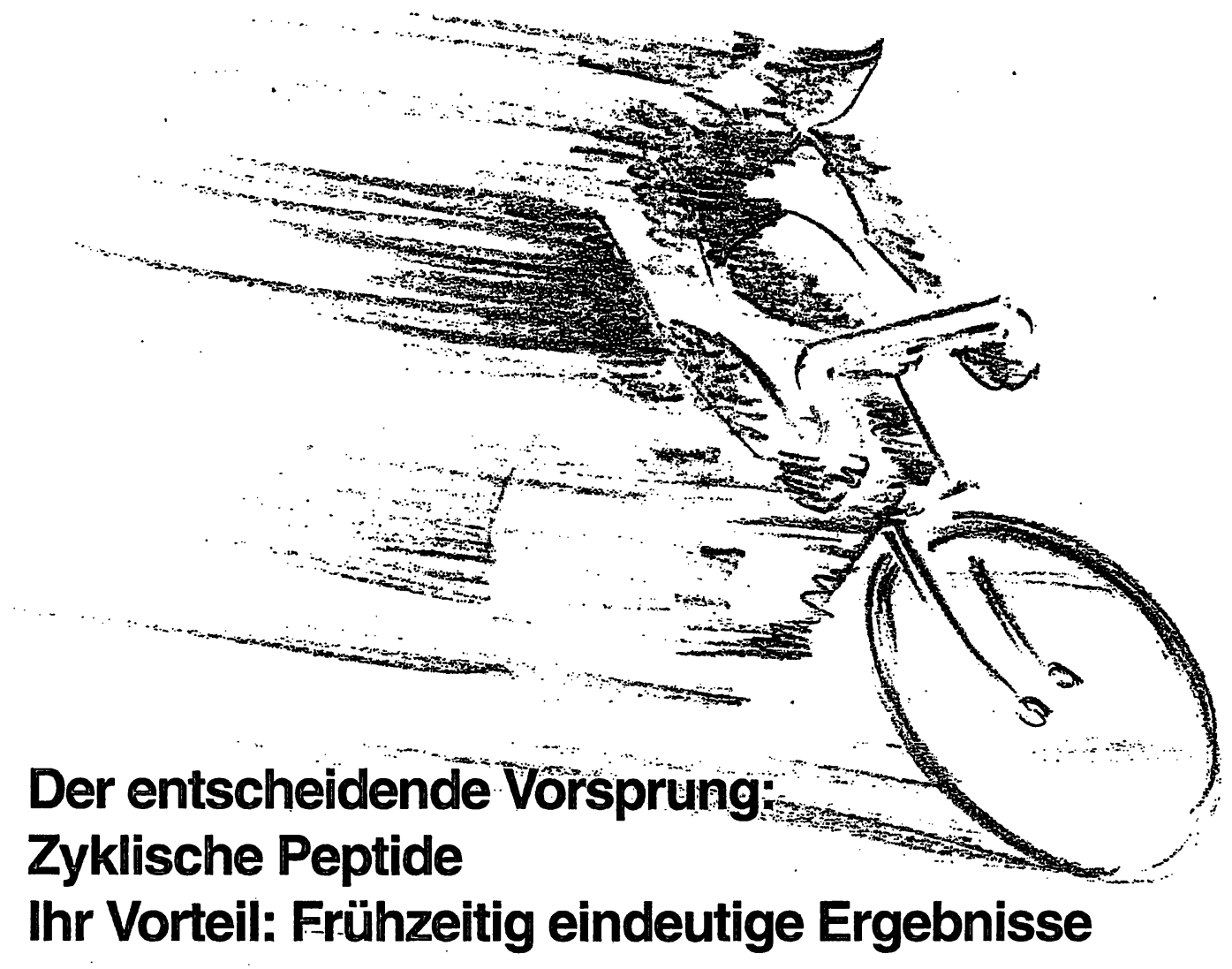




\section{"... eine ähnliche Provokation \\ wie die satanischen Verse"}

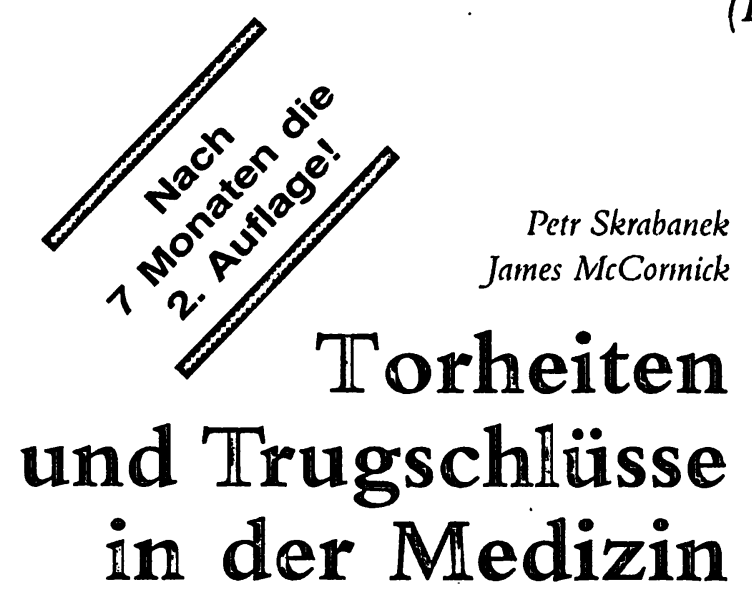

ISBN 3-87409-055-8, 164 Seiten, Fadenheftung, 35, - DM (kongenial ïbersetzt von Dres. Chantelau, Cleveland, Sawicki und Richter, alle Med. Klinik der Heinrich-Heine-Universität, Düsseldorf)
(L'Express)
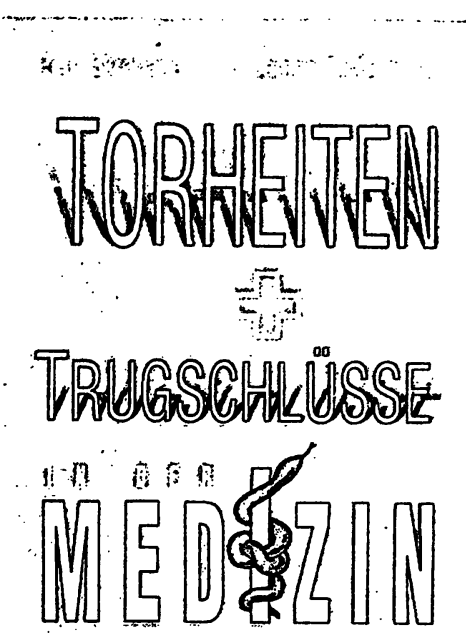

-

\section{Endlich auch in Deutschland:}

"Torheiten und Trugschlüsse in der Medizin" attackiert die Selbstgefälligkeit des zeitgenössischen ärztlichen Denkens und fordert eine rationale Bestandsaufnahme etablierter medizinischer Praktiken.Die Autoren empfehlen Skepsis gegenüber Diagnosen, Behandlungen und Heilmethoden und setzen sich mit angelsächsischem, bisweilen triefend schwarzem Humor mit "Absurditäten und Modeerscheinungen" des Medizinbetriebes auseinander vom Placebo über Homöopathie und Akupunktur bis zur Prävention: "Das Buch ist wichtig für alle, die besser verstehen wollen, was Medizin ist und was nicht" (New England Journal of Medicine zur britischen Originalausgabe).

\section{Verlag Kirchheim, Postfach 25 24, 6500 Mainz}

Ich bestelle ........ Expl. Skrabanek/McCormick, Torheiten und Trugschlüsse in der Medizin, 2. Auflage, ISBN 3-87409-055-8, 35,- DM

Name:

Straße:

$\therefore$

PLZ/Ort:

Datum: Unterschrift: 
Der Schwerpunkt der Labordiagnostik liegt bei den genetisch möglichen Konduktorinnen. Es sind dieses

1. Mütter von 1 hämophilen Sohn ohne bekannte familiäre Hämophilie,

2. Frauen mit hämophilen Verwandten (Schwestern von Hämophilen, Töchter der Schwestern von Hämophilen, Töchter und Schwestern sicherer Konduktorinnen).

Die sogenannte konventionelle Diagnostik von Konduktorinnen der Hämophilie B beruht auf der Bestimmung des Genproduktes des Faktor-IX-Gens als Faktor-IX-Aktivität (IX:C) und Faktor-IX-Antigen (IX-Ag). Sie ist infolge der ausgeprägten Heterogenität der Untersuchungsbefunde erschwert und setzt daher eine Subklassifizierung des bzw. der Hämophilie-Patienten in jeder einzelnen betroffenen Familie voraus (4).

Ziel der Untersuchungen war die Beantwortung der Frage, ob trotz der Möglichkeit einer genomischen Diagnostik eine konventionelle Diagnostik sinnvoll ist.

\section{Patienten und Methodik}

65 Frauen aus 24 Familien mit Hämophilie B wurden mehrfach gerinnungsanalytisch untersucht. Es handelte sich hierbei um 19 genetisch sichere und 46 genetisch mögliche Konduktorinnen.

Schwerpunkte der Untersuchungen bildeten folgende Untersuchungen:

- Faktor-IX-Aktivität (IX:C)

Einstufige Bestimmung mit Reagentien der Boehringer Mannheim GmbH und der Behring AG Marburg

- Faktor-IX-Antigen (IX:Ag)

ELISA der Boehringer Mannheim GmbH

Die Beurteilung der möglichen Konduktorinnen erfolgte unter Berücksichtigung der Ergebnisse bei gesunden Frauen, der Subklasse des/der Hämophilie-B-Patienten der betreffenden Familie und der Ergebnisse bei den genetisch sicheren Konduktorinnen der entsprechenden Subklasse bzw. Familie.

\section{Ergebnisse}

In der Tabelle 1 sind die Ërgebnisse der Bestimmungen von IX: $\mathrm{C}$ und $\mathrm{IX}: \mathrm{Ag}$ sowie die Quotienten aus beiden $\mathrm{Pa}$ rametern bei gesunden Frauen aufgeführt. Bei Frauen, die keine Kontrazeptiva einnahmen, betrugen die Mittelwerte von IX:C und IX:Ag etwa $100 \%$, so daß der Quotient aus beiden Untersuchungsergebnissen nahe 1 lag. Bei Frauen, die orale Kontrazeptiva einnahmen, lagen $\mathrm{IX}: \mathrm{C}$ und $\mathrm{IX}: \mathrm{Ag}$ zwar höher, der Quotient war jedoch ebenfalls etwa 1. Bei Schwangeren im 3. Trimenon kam es zu einer Erniedrigung des Quotienten, bedingt durch die unproportionale Erhöhung von IX:Ag. Tabelle 2 enthält Ergebnisse bei genetisch sicheren Konduktorinnen der verschiedenen Hämophilie-B-Subklassen. In Familien mit Hypo-IX-ämie wurde immer eine gleichsinnige $\mathrm{Er}$ niedrigung von $\mathrm{IX}: \mathrm{C}$ und $\mathrm{IX}: \mathrm{Ag}$ beobachtet, so daß Quotienten um 1 resultierten. Anders verhielten sich die Meßwerte bei sicheren Konduktorinnen aus Familien mit Normodys-IX-ämie. IX:C war disproportional gegenüber $\mathrm{IX}: \mathrm{Ag}$ erniedrigt, so daß die Quotienten stets wesentlich niedriger als 1 lagen. Uneinheitlich waren die Ergebnisse bei den sicheren Konduktorinnen der Hypodys-IX-ämie. Ein Teil dieser Konduktorinnen wies Ergebnisse entsprechend den Konduktorinnen der Hypo-IX-àmie auf, der an-
Tab. 1: IX: C und IX:Ag bei gesunden Frauen

\begin{tabular}{|c|c|c|c|c|}
\hline & \multirow{3}{*}{$\mid \mathrm{X}: \underset{\bar{x}}{\mathrm{C}}(\%)$} & \multirow{3}{*}{$\mid X: \underset{\bar{x}}{\operatorname{Ag}(\%)}$} & \multicolumn{2}{|c|}{$\mid X: C(\%)$} \\
\hline & & & $I X: A$ & $g(\%)$ \\
\hline & & & $\bar{x}$ & $\overline{\mathrm{x}} \pm 2,3 \mathrm{~s}$. \\
\hline $\begin{array}{l}\text { Ohne Kontrazeptiva } \\
(n=25)\end{array}$ & 98,9 & 96,4 & 1,03 & $0,83-1,22$ \\
\hline $\begin{array}{l}\text { Mit Kontrazeptiva } \\
(n=25)\end{array}$ & 113,5 & 115,0 & 0,98 & $0,84-1,13$ \\
\hline $\begin{array}{l}\text { Schwangere, 3. Trimenon } \\
(n=25)\end{array}$ & 120,2 & 151,9 & 0,79 & $0,65-0,94$ \\
\hline
\end{tabular}

dere Teil dagegen entsprechend den Konduktorinnen der Normodys-IX-ämie.

Eine Zusammenstellung der Quotienten aller 19 untersuchten sicheren Konduktorinnen enthält die Tabelle 3 . Die Ermittlung eines Konduktorinnenstatus bei genetisch möglichen Konduktorinnen der verschiedenen Subklassen wird durch die Stammbäume der Abbildungen 1-3 veranschaulicht. Grundlage der Zuordnung zu einem Konduktorinnenstatus waren die an sicheren Konduktorinnen für den jeweiligen Subtyp ermittelten Werte.

Tab. 2: Ergebnisse bei genetisch sicheren Konduktorinnen der Hämophilie B

\begin{tabular}{|c|c|c|c|}
\hline \multirow{3}{*}{ Konduktorin } & \multicolumn{3}{|c|}{ 1. Hypo-IX-ämie } \\
\hline & $I X: C(\%)$ & $\mathrm{IX}: \mathrm{Ag}(\%)$ & $\mathrm{IX}: \mathrm{C}(\%)$ \\
\hline & & & $\overline{I X: A g(\%)}$ \\
\hline 1 & 20 & 22 & 0,91 ， \\
\hline 2 & 30 & 32 & 0,94 \\
\hline 3 & 41 & 38 & 1,08 \\
\hline 4 & 51 & 60 & 0,85 \\
\hline
\end{tabular}

\begin{tabular}{|c|c|c|c|}
\hline \multirow{3}{*}{ Konduktọrin } & \multicolumn{2}{|c|}{ 2. Normodys-IX-ämie } & \multirow{3}{*}{$\frac{\mid X: C(\%)}{I X: \operatorname{Ag}(\%)}$} \\
\hline & $\mathrm{IX}: \mathrm{C}(\%)$ & IX:Ag (\%) & \\
\hline & & & \\
\hline 1 & 21 & 95 & 0,22 \\
\hline 2 & 31 & 77 & 0,40 \\
\hline 3 & 62 & 109 & 0,57 \\
\hline 4 & 82 & 168 & 0,49 \\
\hline
\end{tabular}

\begin{tabular}{lccc}
\hline \multirow{2}{*}{ Konduktorin } & \multicolumn{3}{l}{ 3. Hypodys-IX-ämie } \\
& $\mathrm{IX}: \mathrm{C}(\%)$ & $\mathrm{IX}: \mathrm{Ag}(\%)$ & $\frac{\mathrm{IX}: \mathrm{C}(\%)}{\mathrm{IX:Ag}(\%)}$ \\
\hline 1 & 38 & 45 & 0,84 \\
2 & 52 & 61 & 0,85 \\
\hline 3 & 21 & 53 & 0,40 \\
4 & 47 & 100 & 0,47 \\
\hline
\end{tabular}

Tab. 3: IX: C/IX:Ag bei sicheren Konduktorinnen der Hämophilie B

\begin{tabular}{lcc}
\hline & \multicolumn{2}{c}{$\mathrm{IX:C( \% )}$} \\
\cline { 2 - 3 } & \multicolumn{2}{c}{$\overline{\mathrm{IX}: \mathrm{Ag}(\%)}$} \\
\hline 1. Hypo-IX-ämie & 0,93 & $\overline{\mathrm{x}} \pm 2,3 \mathrm{~s}$ \\
2. Normodys-IX-ämie & 0,44 & $0,19-1,16$ \\
3. Hypodys-IX-ämie & & \\
Gruppe 1 & 0,88 & $0,70-1,06$ \\
Gruppe 2 & 0,51 & $0,21-0,81$ \\
\hline
\end{tabular}

Lab.med. 16: 243 (1992) 
In der Abbildung 1 ist eine Familie mit Hypo-IX-ämie dargestellt. Dic vier Frauen der dritten Generation sind mög. liche Konduktorinnen der Hämophilie B. Bei zwei von ihnen (III 1 und III 4) konnte ein Konduktorinnenstatus bestätigt werden. In ăhnlicher Weise war auch eine Bestätigung bzw. ein wahrscheinlicher Ausschluß eines Konduktorinnenstatus in Familien mit Normodys-IX-ämie (Abb. 2, I 2 und || 3) und Hypodys-IX-ämie (Abb. 3, II 1 und III 1) möglich.

\section{Diskussion}

Versuche zur gerinnungsanalytischen Identifizierung von Konduktorinnen der Hämophilie $B$ begannen mit Bestimmungen der Faktor-IX-Aktivität und des Faktor IX CRM (cross-reacting material) (3). Quotientenbildungen aus $\mathrm{IX}: \mathrm{C}$ und $\mathrm{IX}: \mathrm{Ag}$ lieferten die ersten brauchbaren Ergeb- nisse $(5,6)$. Sie wiesen ausgeprägte Unterschiede aufgrund der Heterogenität der Hämophilie B mit daraus resultierenden Interpretierungsschwierigkeiten auf. Diese Tatsache sowie die Seltenheit des Krankheitsbildes und Unzulänglichkeiten der Bestimmungsmethoden (7) waren die Ursache, daß eine auf gerinnungsanalytischen Methoden beruhende Konduktorinnendiagnostik der $\mathrm{Hä-}$ mophilie B kaum in größerem Umfang durchgeführt wurde.

Das von uns praktizierte Vorgehen hat eine Subklassifizierung der betreffenden Hämophilie-B-Familie zur Voraussetzung (4) und sollte nach Möglichkeit eine Untersuchung von sicheren Konduktorinnen, vor allem bei dem Subtyp Hypodys-IX-ämie, einschließen. Eine Wiederholungsuntersuchung ist empfehlenswert.

Die Laboruntersuchungen, die im wesentlichen auf einer einstufigen Faktor-IX-Aktivitätsbestimmung und einer

Abb. 1: Hypo-1X-ämie (Stamm B./R.)

a Hämophilie-B-Patient

- Hämophilie-B-Konduktorin

0 Gesunde Frau

Zahlenwerte: IX:C (\%)/IX:Ag (\%)

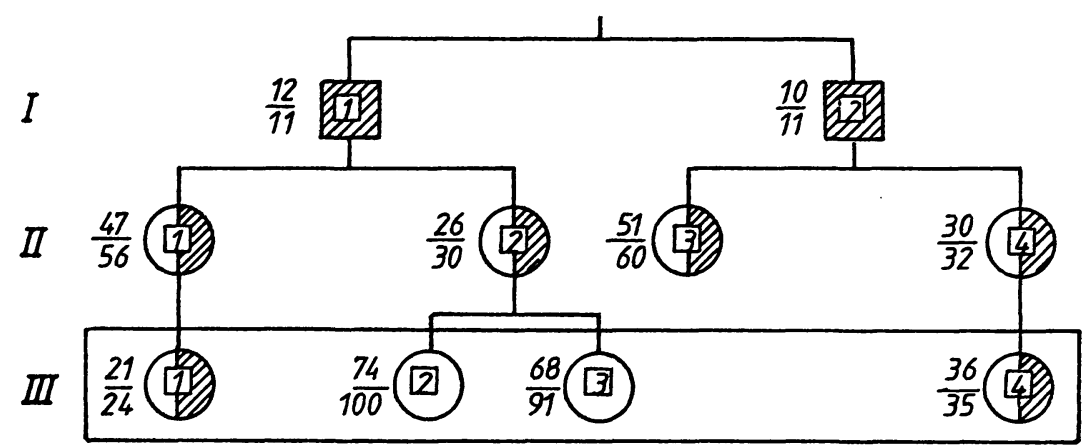

Abb. 2: Normodys-IX-ämie (Stamm F.) Erläuterungen s. Abb. 1

Abb. 3: Hypodys-IX-ämie (Stamm Sch./R.) Erläuterungen s. Abb. 1 .
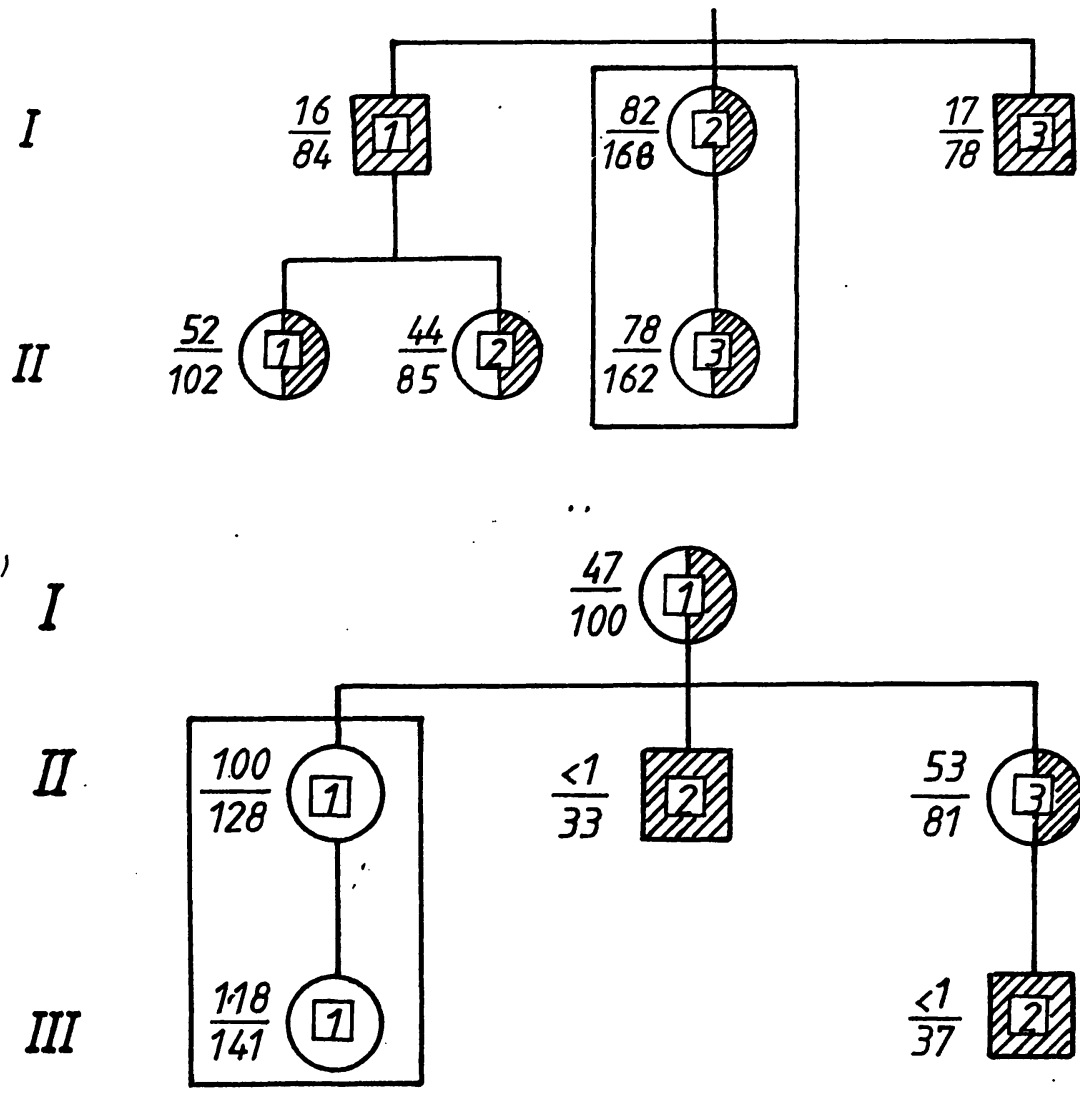
Faktor-IX-Antigenbestimmung mittels Elisa beruhen, sind schnell und problemlos durchführbar. Bei gesunden Frauen lagen die Quotienten aus IX:C und IX:Ag um 1, auch bei Einnahme oraler Kontrazeptiva, da sich beide Parameter gleichsinnig erhöhen. In Abhängigkeit vom Subtyp der Hämophilie B waren bei genetisch sicheren Konduktorinnen die Absolutwerte von IX:C und IX:Ag sowie die Quotienten unterschiedlich. Dieses Verhalten diente als Ausgangs- bzw. Vergleichsbasis für die Beurteilung genetisch möglicher Konduktorinnen.

Mit Hilfe gerinnungsanalytischer Untersuchungen können nicht mehr als $80 \%$ der Hämophilie-B-Konduktorinnen identifiziert werden (8). Wesentliche Fortschritte wurden mittels Molekulargenetik und genomischer Diagnostik erreicht. Nachdem 1983 erstmals mit gentechnologischen Methoden ein Defekt bei der Hämophilie B nachgewiesen werden konnte (9), erfolgten kurz danach Untersuchungen bei Konduktorinnen $(10,11)$. Seither ist eine große Anzahl von Ergebnissen bekanntgeworden, die die Vorteile bei der Carrier- und pränatalen Diagnostik belegen $(8,12,13)$. Jedoch erlauben auch diese Methoden keine absolut sichere Aussage.

Wir sind der Auffassung, daß auf eine konventionelle Konduktorinnendiagnostik nicht verzichtet werden sollte. Sie müßte der genomischen Diagnostik vorgeschaltet sein, da sie einfach durchführbar ist und bei vielen Frauen bereits zum Ergebnis führt. Bei einer Reihe der genetisch möglichen Konduktorinnen der Hämophilie B ist wie bei der Hämophilie A keine sichere Aussage möglich. Bei diesem Personenkreis ist eine genomische Diagnostik unumgänglich.

Die Ermittlung gerinnungsphysiologischer Laborwerte im Sinne einer konventionellen Diagnostik ist somit als sinnvolle Ergänzung zu den molekulargenetischen Methoden zu betrachten. Diese Feststellung, die durch Oldenburg et al. (14) bei der Konduktorinnendiagnostik der Hämophilie A getroffen wurde, hat in gleicher Weise auch für die Hämophilie B Berechtigung.
Schriftum:

1. Colman, R. W., Hirsh, J., Marder, V. J., Salzman, E. W. (1982) Hemostasis and Thrombosis. J. B. Lippincott, Philadelphia, Toronto.

2. Williams, W. J., Beutler, E., Erslev, A. J., Lichtman, M. A. (1983) Hernatology. Mc Graw-Hill Book Company.

3. World Health Organization (1977) Methods for the detection of haemophilia carriers: a memorandum. Bull. WHO 55, 675 .

4. Lutze, G., Hartung, K.-J. Presser, H.J., Urbahn, H. Konventionelle Labordiagnostik der Hămophilie B, I. Subklassifizierung der Hämophilie B. Lab.med. (in Vorbereitung) 5. Pechet, L., Tiarks, C. Y., Stevens, J., Sudhindra, R. R., Lipworth, L. (1978) Relationship of factor IX antigen and coagulant in haemophilia $B$ patients and carriers. Thrombos. Haemostas. 40, 465.

6. Orstavik, K. H., Veltkamp, J. J., Bertina, R. M., Hermans, J. (1979) Detection of carriers of haemophilia B. Brit. J. Haematol. 42, 293.

7. Lipworth, L., Tiarks, C. Y., Rice, C. A., Pechet, L. (1979) Laboratory prediction of the 7. Lipworth, L., Tiarks, C. Y., Rice, C. A., Pechet, L. (1979) Laboratory prediction of the
carrier state in hemophilia B. Role of replication of assays. Amer. J. Clin. Pathol. 72, carrier
1000 .

8. Wehnert, M., Herrmann, F. H., Wulff, K. (1988) Zur molekulargenetik und genomischen Diagnostik (Carrierdiagnostik und pränatale Diagnostik) bei Hämophilie $B$. Z. klin. Med. 43, 2195.

9. Gianelli, F., Choo, K. H., Rees, D. J. G., Boyd, Y., Rizza, C. R., Brownlee, G. G. (1983) Gene deletions in patients with haemophilia $B$ and antifactor IX antibodies. Nature 303, 181.

10. Gianelli, F., Choo, K. H., Winship, P. R., Rizza, C. R., Anson, D. S., Rees, D. J. G., Ferrari, N., Brownlee, G. G. (1984) Characterization and use of an intragenic polymorphic marker for detection of carriers of haemophilia B. Lancet 1,239

11. Grunebaum. L., Cazenave, J. P., Camerino, G., Kloepfer, C., Mandel, J. L. (1984) Carrier detection of hemophilia $\mathrm{B}$ by using a restriction site polymorphism associated with the coagulation factor IX gene. J. Clin. Invest. $73,1491$.

12. Ludwig, M., Schwaab, R., Oldenburg, J., Olek, K., Brackmann, H.-H. (1990) Konduk torinnen-Diagnostik und molekulare Grundlagen der Hāmophilie. 20. Hămophilie Symposium Hamburg 1989 (Hrsg.: Landbeck, G., Marx, R., Scharrer, I., Schramm, W.) Springer-Verlag, Berlin, Heidelberg.

13. Zoll, B., Knobloch, O., Hilker, A. (1990) Molukulargenetische Analysen der Hämo13. Zoll, B., Knobloch, O., Hilker, A. (1990) Molukulargenetische Analysen der Hämo-
philie A und B. 20. Hämophilie-Symposium Hamburg 1989 (Hrsg.: Landbeck, G., Marx, R., Scharrer, I., Schramm, W.) Springer-Verlag, Berlin - Heidelberg.

14. Oldenburg, G. J., Schwaab, R., Hammerstein, U., Ludwig. M., Olek, K., Brackmann, H.-H. (1991) Konduktorinnendiagnostik in der Hämophilie A - eine Strategie zur Einbeziehung gerinnungsphysiologischer Laborwerte als sinnvolle Ergänzung zu den molekulargenetischen Methoden. 21. Hämophilie-Symposium Hamburg 1990 (Hrsg.: Landbeck, G., Scharrer, I., Schramm, W.) Springer-Verlag, Berlin, Heidelberg.

Anschrift der Verfasser:

Dr. med. habil. G. Lutze

Dr. rer. nat. K.-J. Hartung

Prof. Dr. rer. nat. habil. H. Urbahn

Institut für Klinische Chemie und Laboratoriumsdiagnostik der Medizinischen Akademie

Leipziger Straße 44

0-3090 Magdeburg

Dr. med. H.-J. Presser

Walther-Friedrich-Krankenhaus

Birkenallee 34

0-3039 Magdeburg 